Recepción: 21 / 06 / 2017

Aceptación: 22 / 07 / 2017

Publicación: 15/ 11/ 2017
Ciencia de la salud

Artículo de investigación

\title{
Clínica psicoanalítica para pacientes con cáncer
}

\author{
Psychoanalytic Clinic for Cancer Patients
}

\section{Clínica psicoanalítica para pacientes com câncer}

Inés Vera-Molina ${ }^{\mathrm{I}}$

ines.vera@uleam.edu.ec

Amira Trujillo-Eljuri II

amira.trujillo@uleam.edu.ec

Correspondencia: ines.vera@uleam.edu.ec

I Magister en Psicoanálisis con Mención en Clínica Psicoanalítica, Psicóloga Clínica, Docente en la Universidad Laica Eloy Alfaro de Manabí, Manta, Ecuador.

II Magister en Educación Superior, Diploma Superior en Educación Superior, Psicólogo Clínico, Docente en la Universidad Laica Eloy Alfaro de Manabí, Manta, Ecuador. 


\section{Resumen}

Esta investigación se llevó a cabo en el marco de la obtención del título de Magister en Psicoanálisis con mención en clínica Psicoanalítica. La misma responde determinadas preguntas de investigación: ¿El psicoanálisis puede ofrecer su trabajo para la contención de los sujetos con cáncer? ¿Qué modos de intervención se pueden ubicar desde la clínica psicoanalítica? Este estudio se realiza en el Hospital Oncológico "Julio Villacreses Colmont", Solca Manabí, de Portoviejo. Luego de haber sido diagnosticados con cáncer, los pacientes deben asistir a la consulta psicológica, por lo 'traumático' que puede ser el recibir esta noticia. Hay sujetos que se colocan en una posición de negación de la realidad, otros presentan cuadros depresivos con una presencia importante de angustia. Un diagnóstico de cáncer provoca en el sujeto una conmoción intensa en su subjetividad, que al no ser capaz de resolverlos por sus medios habituales provocará distintas respuestas. Es a partir de esta situación que los casos remitidos fueron atendidos y conceptualizados desde el psicoanálisis.

Palabras clave: pacientes con cáncer; psicoanálisis; diagnóstico oncológico; clínica psicoanalítica.

\section{Abstract}

This research was carried out in the framework of obtaining the title of Magister in Psychoanalysis with mention in clinical Psychoanalytic. It answers certain research questions: Can psychoanalysis offer its work for the containment of subjects with cancer? What modes of intervention can be found from the psychoanalytic clinic? This study is carried out at the Oncology Hospital "Julio Villacreses Colmont", Solca Manabí, in Portoviejo. After being diagnosed with cancer, patients should attend the psychological consultation, because of the 'traumatic' that may be the receiving of this news. There are subjects who are placed in a position of denial of reality, others present depressive pictures with a significant presence of distress. A diagnosis of cancer causes in the subject an intense commotion in his subjectivity, that not being able to solve them by his habitual means will provoke different answers. It is from this situation that the referred cases were attended and conceptualized from psychoanalysis.

Keywords: cancer patients; psychoanalysis; oncological diagnosis; psychoanalytic clinic. 


\section{Resumo}

Esta pesquisa foi realizada por um cabo no marco da obtenção do título de Magister em Psicoanálisis com menção em clínica Psicoanalítica. A mesma resposta às necessidades de investigação: ¿O psicoanálisis pode oferecer o trabalho para a contenção dos sujeitos com o cancer? ¿Qué modos de intervenção se podem colocar a partir da clínica psicoanalítica ?. Este estudio é realizado no Hospital Oncológico "Julio Villacreses Colmont", Solca Manabí, de Portoviejo. Depois de ter sido diagnosticados com câncer, os pacientes devem ser vistos na consulta psicológica, por lo 'traumático' que pode ser recebido esta noticia. Os sujeitos que se colocam em uma posição de negação da realidade, outros apresentam cadros depresivos com uma presença importante de angustia. Um diagnóstico de câncer provoca no sujeito a uma conmoção intensa em su subjetividad, que não é capaz de resolverlos por sus meios habituales provocará respostas diferentes. É a partir de esta situação e os casos remitidos e atendidos e conceituados a partir do psicoanálisis.

Palavras chave: pacientes con cancer; psicoanálisis; diagnóstico oncológico; clínica psicoanalítica.

\section{Introducción}

El cáncer es una enfermedad que desde el siglo XIX, ha despertado interés en el mundo científico incluyendo las respuestas terapéuticas para encontrar la posible cura.

Según las estadísticas que recoge el Departamento de Registro del Hospital Oncológico "Julio Villacreses Colmont", Solca Manabí, de Portoviejo, en el 2011, alrededor de 800 nuevos pacientes se diagnosticaron con cáncer y fueron atendidos a través de 1530 consultas psicológicas, entre las de primera vez y las consecutivas. El más frecuente de los tipos de cáncer en las mujeres es el de cérvix y en los hombres de próstata, sin embargo, no deja de ser considerable el alto crecimiento de cáncer de mama en la población, el cual ocupa el segundo lugar dentro de las estadísticas nacionales, seguidos de los canceres linfáticos.

Solamente el hecho de mencionar esta enfermedad, provoca en muchos sujetos angustia por su significación enlazado a la muerte. 
Independientemente de la gravedad de la enfermedad y del tratamiento a indicar en el paciente oncológico, están presentes múltiples angustias, entre ellas, angustias de tipo narcisistas como la caída del pelo, el adelgazamiento, el subir de peso, el cambio de color de la piel y la angustia a la mutilación corporal. Respecto a la enfermedad misma, observamos en pacientes oncológicos angustias referentes a que la enfermedad avance hasta convertirse en metástasis, al dolor, al mal resultado de las quimioterapias y a la posibilidad de morir. Respecto a la relación con los otros son comunes angustias, como un empeoramiento de la relación con la pareja, a no poder trabajar más o a no poder estar más para la familia, a estar socialmente aislado, a necesitar continuamente cuidados y ser una carga para los demás. Finalmente, de acuerdo a lo observado, a lo anterior, se añaden angustias de tipo institucionales como la angustia de no ser atendidos por el médico, etc. (Sotelo, 2014).

Más allá de los obstáculos y/o resistencias que encontramos en el camino que conduce a un paciente oncológico hacia el psicoterapeuta, y aun cuando no se haya probado científicamente la asociación entre la asistencia psicológica y una mayor sobrevida, algunas investigaciones muestran que sí se asocia a una mejor calidad de vida, careciendo de los efectos adversos que a veces poseen los tratamientos médicos. $\mathrm{Y}$ en algunos casos también coexiste con estados de remisión que la ciencia tampoco puede explicar. (Chab, 2006).

Desde el 2011, el Departamento de Psicología del Hospital Oncológico "Julio Villacreses Colmont", Solca Manabí, de Portoviejo, pasó a ser un servicio de la consulta externa. Las psicólogas que laboran en la esta institución poseen distintos criterios en relación al tratamiento psicológico a realizar en la atención con pacientes oncológicos. Los diagnósticos psicopatológicos se basan en el Manual de Diagnóstico de Salud, DSM IV, exigencia de los diferentes departamentos de Psicología o Salud Mental de las Instituciones regidas por el Ministerio de Salud Pública del Ecuador. En este momento no se cuenta con la atención de un médico psiquiatra, como sucede en otras organizaciones con la misma misión estratégica. Cuando, por sugerencia del servicio o/y por criterio médico, se hace necesario la medicación psicofarmacológica, el paciente es derivado al Médico Internista para su respectiva evaluación.

Los pacientes son atendidos en el área de consulta externa, quimioterapia y hospitalización, cuya demanda parte, en algunas ocasiones, del médico tratante, el cual cumple muchas veces con lo 
establecido en los protocolos de atención en consulta externa y hospitalización o en algunas ocasiones las derivaciones provienen bajo pedido del paciente.

Este trabajo está basado en torno a la demanda de ayuda psicológica de los sujetos con cáncer en consideración a su sentir en relación a la enfermedad y al criterio de algunos médicos, que consideran que hay algo más allá de los puntos estrictamente científicos. Por tanto, este trabajo tiene como preguntas de investigación

- ¿Qué hacer para mejorar la condición y calidad de vida de los pacientes frente al diagnóstico y al tratamiento?

- ¿El psicoanálisis puede ofrecer su trabajo para la contención de los sujetos con cáncer?

- ¿Qué modos de intervención se pueden ubicar desde la clínica psicoanalítica?

\section{Metodología}

Es un estudio no experimental, cualitativo y descriptivo. Se procedió a la revisión de la historia clínica de los pacientes, para realizar la identificación de los casos (no antecedentes de cuadros psicóticos ni depresivos graves, temprana detección del cáncer y con performance hasta del $60 \%$ ). Se llevaron a cabo 15 entrevistas semi-estructuradas y abiertas, que iniciaron desde la detección del diagnóstico. Se trabajó con 7 casos clínicos, ya que existía predisposición al trabajo (demanda de ayuda). Todos los pacientes que llegaban a consulta fueron atendidos desde esta posición clínica psicoanalítica, pero algunos no cumplían con los criterios y otros dejaban las consultas y optaron por la eventualidad de la atención.

Luego de las 15 entrevistas se realizaron otras de control o acompañamiento, es decir que, si no existía la derivación por la demanda de saber, se siguió atendiendo al sujeto de forma paulatina (el paciente demandaba consulta), cuando existía eventos contingentes como la reactivación de enfermedad o de la cotidianidad.

Se revisaron los antecedentes teóricos sobre el tema, a través de la búsqueda en las bases de datos de revistas indexadas y no indexadas, por ser un tema que contaba al momento con información sobre temas en atención a pacientes con cáncer o el psicoanálisis en pacientes con cáncer. 
Así mismo se buscó sobre otras modalidades de trabajo terapéutico, desde otras perspectivas teóricas: sistémica, cognitivo-conductual, humanista.

Se trabajó además con especial interés los discursos psicoanalítico y médico pues tratar estos temas de manera conjunta supondría suscitar los habituales problemas entre las palabras y las cosas. Al hacerse esta investigación en una institución hospitalaria, no se pudo dejar de relacionar y diferenciar qué aportan cada uno de ellos en la mejoría de los pacientes diagnosticados con cáncer. El discurso es un lazo social basado en el lenguaje y que se articula sobre una praxis.

El discurso médico pone en evidencia ciertos hechos, articularlos en la lógica que le es propia e impone cierta mirada en el dominio por él constituido. Es una disciplina de evaluación de los hechos, e impone ésta a quien sigue su discurso. "Porque lo que el médico observa es lo que puede inscribirse en cierto campo de saber, con exclusión de cualquier otra cosa; es lo que puede constituir una teoría, o al menos un diagnóstico, que tenga alguna coherencia, el resto no existe para él" (Clavreul, J. 1978).

El discurso del analista se coloca desde el principio en la particularidad, en lo singular de cada subjetividad, en lo que tiene de inédito el discurso de cada sujeto, el deseo de cada cual. Es que, para el psicoanálisis, la realización de un abordaje representa la singularidad que se le da a cada sujeto. Lacan (1950), plantea que el discurso del analista debe encontrarse en el punto opuesto a toda voluntad de dominar aclarando que es fácil deslizarse de nuevo hacia el discurso del dominio.

En la revisión final se presentaron 3 casos clínicos, desde el dispositivo psicológico de orientación psicoanalítica.

\section{Desarrollo}

Los psicólogos clínicos de orientación psicoanalítica insertados en una institución hospitalaria, tienen un compromiso más allá del tiempo, del sueldo y de los semblantes hospitalarios. Es una clínica que tiene que ver con el saber-hacer, con el saber-escuchar, con el saber-decir, tanto del psicólogo clínico como de los pacientes; es decir una ética que concierne un trabajo con el sufrimiento humano. 
Se considera a la clínica psicoanalítica que se diferencia de la clínica médica en la manera de intervenir con el 'enfermo', por ejemplo, el psicoanálisis comienza cuando hay transferencia, ahí donde se detiene la clínica médica. (Vera Molina, 2013).

Frente al diagnóstico de cáncer (relacionado con la significante muerte) ocurre en los sujetos una ruptura, una ambivalencia, una división que deja al deseo en tensión. Hay que estar prestos a escuchar más allá de lo que el paciente plantea, tener en cuenta la posición que haya tomado frente a la enfermedad y el tratamiento, porque es ahí que se pone en juego el deseo que habita al paciente.

Considerando las reflexiones freudianas y el trabajo con los sujetos en esta investigación, podemos pensar que los sujetos que sufren esta enfermedad evidencian una experiencia de duelo. La clínica psicoanalítica apunta en estos casos a un trabajo de simbolización.

El psicoanálisis no apunta a perturbar y mucho menos a borrar este dolor que invade al sujeto, más bien postula al duelo como 'resorte fundamental de la constitución del deseo', como momento subjetivante. Un duelo es ese momento singular para cada sujeto.

Se coincide que en la práctica hospitalaria, el psicoanálisis se presenta como un ámbito de cruces de discursos, un ámbito de condensación de demandas de muy diverso tipo y origen y, correlativamente, de prácticas muy diversas, agrupadas bajo el común denominador de lo médico. El discurso psicoanalítico se muestra como una modalidad particular de trabajo, frente a las urgencias que se presentan en la práctica a diario y que demanda respuestas rápidas.

Prácticamente toda la constelación de fenómenos que aparecen en la urgencia subjetiva, pueden reducirse a dos órdenes clínicos en la estructura: la certeza y la angustia. La certeza situada en su costado más enigmático como son los fenómenos de significación personal, de xenopatía en el cuerpo y en el pensamiento y la perplejidad misma, es decir fenómenos pre-delirantes. La angustia, como acontecimiento, como trauma y sus versiones: el pánico, el vértigo, entre otros, presentifican la pérdida de la topografía imaginaria que organiza al sujeto. 
Los síntomas presentados en la consulta de urgencia o en la primera entrevista del proceso de interconsulta, que se manejan en la consulta psicológica de la institución, suelen ser claramente identificables dentro de los cuadros descritos por la psiquiatría clásica o por el DSM.

A través de la escucha atenta, la persona que padece angustia irá expresando sus sufrimientos, malestares y temores que tienen que ver con lo vivido en el diagnóstico, tratamiento médico realizado y su situación actual. El psicoanalista debe propiciar un lugar para el habla, facilitando la apertura a un espacio discursivo a través de la palabra y la contención emocional, la historia de la enfermedad, los sueños, las angustias, etc. El psicoanalista debe comprender el sufrimiento del paciente oncológico. El poder expresar aquello de lo que lo aqueja, previene y reduce la intensidad de la angustia haciéndola más llevadera. En la interpretación subjetiva de la angustia física y emocional que manifiesta el paciente estando en la etapa de remisión de su cáncer y en la puesta en marcha de mecanismos defensivos funcionales, para que el yo sea capaz de obtener herramientas para continuar su vida cotidiana o enfrentar nuevamente un tratamiento médico. (Sotelo, 2014).

En la primera consulta se indaga en el paciente el sentimiento subjetivo de sufrimiento y se aborda cada caso animado por una 'docta ignorancia', ya que la urgencia obliga al analista a ejercitarse aún más en esa disciplina del 'olvidar lo que sabe' y la urgencia en si supone ya una intervención analítica, es decir, implica que en el dispositivo en el cual se acoge la demanda del que sufre hay ya un practicante del psicoanálisis que apunta a situar cuál es la respuesta singular de ese sujeto, es decir, cuál es el sujeto que va a producir una respuesta frente ese encuentro con la muerte.

Se puede decir que la clínica de la urgencia subjetiva es, en tanto tal, una clínica que lleva al máximo la exigencia analítica de escuchar el detalle del discurso de aquel que consulta. Ricardo Seldes (2004), define a la clínica de la urgencia como "ruptura de la cadena significante, ya que si el significante no se articula el sujeto no puede representarse y cuando esto ocurre el sujeto apela a los recursos que la ausencia de lenguaje hace brotar, el actingout o el pasaje al acto". 


\section{Conclusiones}

A partir de los enunciados referidos, es posible trabajar con una perspectiva de orientación lacaniana en la atención a pacientes oncológicos, dando un espacio para que el sujeto que demande atención o sea derivado, tenga la oportunidad de replantear su posición frente a la enfermedad y tratamiento, y que asuma una posición de responsabilidad, dando un lugar al deseo.

No es análisis, sino la instauración de un dispositivo de 15 entrevistas, desde la posición psicoanalítica, que dé apertura a la interpelación, posibilidad diagnóstica y realizar la derivación.

El psicoanálisis ofrece un espacio de escucha para el paciente y su familia, se da lugar a la palabra, a los significantes que envuelven este encuentro con lo real para cada sujeto desde su particularidad.

La tarea realmente es la de relanzar el discurso que nos presenta el paciente. Tanto para Freud (1913) como Lacan (1960) hay razones diagnósticas para establecer este tiempo previo al análisis, ya que es el momento en que el sujeto comprende su implicación en la responsabilidad de sus síntomas y el momento de transformar las entrevistas, en análisis propiamente dicho.

\section{Referencias bibliográficas}

Chab, S. (2006) Psicoanálisis y cáncer. Recuperado el 13 de febrero de 2016, de http://www.socargcancer.org.ar/actividades_cientificas/2006_psyco_y_cancer.pdf

Clavreul J. (1978). Citado por: Vera Molina, I. (2013) Clínica psicoanalítica para pacientes con cáncer. Tesis para optar por el Grado Académico de Magíster en Psicoanálisis con mención en Clínica Psicoanalítica.

Freud S. (1913). Sobre la iniciación del tratamiento (Nuevos consejos sobre la técnica del psicoanálisis). Argentina: AMORRORTU EDITORES: AMORRORTU EDITORES

Lacan J. (1950). Psicoanálisis y medicina en intervenciones y textos. España: Edición Manantial

Lacan J. (1959). La ética del psicoanálisis Argentina: Editorial Paidós. Argentina: Paidós 
Seldes R. (2004). Citado por: Vera Molina, I. (2013) Clínica Psicoanalítica para pacientes con cáncer. Tesis para optar por el Grado Académico de Magíster en Psicoanálisis con mención en Clínica Psicoanalítica

Sotelo, C (2014). La angustia de Damocles: Contribuciones al trabajo de la angustia de recaída en pacientes con cáncer a través de la psicoterapia breve de orientación psicoanalítica a propósito de un caso clínico. Tesis para optar al Grado de Magíster en Psicología Clínica de Adultos

Vera Molina, I. (2013). Clínica Psicoanalítica para pacientes con cáncer. Tesis para optar por el Grado Académico de Magíster en Psicoanálisis con mención en Clínica Psicoanalítica 